\title{
AVALIAÇÃO QUANTITATIVA DE SERVIÇOS DE PRÉ-NATAL
}

\section{RSPSP-149}

CIARI $\mathrm{J}_{\mathrm{R} .,}$ C. et al. - Avaliação quantitativa de serviços de pré-natal. Rev. Saúde públ., S. Paulo, 6:361-70, 1972.

Resumo: São propostos 6 itens de avaliação quantitativa de atividades de serviços de Pré-Natal. São referidos, como indicadores de atividades dos médicos e grau de proteção oferecida às gestantes: 1) a média de consultas por hora/ano por médico; 2) o número de consultas possíveis; 3) a média de consultas por gestante; 4) a proporção entre matrículas e consultas em continuação por mês; 5) média de idade da gestação na matrícula. Procurou-se relacionar estes dados com a qualificação apresentada pelos serviços, ou seja, presença de médico, enfermeira, facilidades para internação, laboratório, medicamentos, cursos e assistência social. Estes indicadores parecem revelar, quando associados, não so a quantidade de serviços prestados, como também indiretamente a sua qualificação. Foram observados 10 serviços de Pré-Natal durante 4 anos de atividades, estas avaliadas pelos indicadores propostos. Revelou-se a predominância de baixa produtividade na maioria deles, com excessivo numero de horas ociosas, bem como, número pequeno de consultas para as matriculas. A relação presumida entre qualificação de serviços e maior frequiência ao pré-natal, nấo se revelou clara a não ser quando oferecida intensamente.

UNITERMos: Assistência pré-natal *; Gravidea *
Cyro CIARI Jr. *

Jair L. F. SANTOS **

Pedro Augusto Marcondes de ALMEIDA *

A prestação de serviços em pré-natal tem sido medida apenas pelo número de consultas subsequientes recebidas, crianração se efetivamente elas atingiram o grupo gestante e quanto se aproximaram do esperado em atividades.

Ao lado da qualidade da consulta, o importante, para determinar o grau de proteção recebida pela grávida, é a idade da gestação em que a paciente iniciou o seu atendimento e o número de consultas subsequientes recebidas, criando assim uma possibilidade maior de ter suas complicações diagnosticadas e controladas. As matrículas tardias, mesmo tendo consultas frequientes, apenas atuam sobre processos que por sua gravidade são evidentes. Contudo, os desvios da normalidade que demandam mais tempo para serem identificados e corrigidos, não encontram possibilidade de correção.

Por outro lado, a escassez de profissionais qualificados obriga a uma distribuição de seu tempo de trabalho no sentido de evitar-se as horas ociosas e assim cobrir a crescente demanda de serviços de Saúde Materna. Os níveis de mortalidade peri-natal 1 estão demonstrando a necessidade de ampliação des-

* Da Disciplina de Higiene Materna do Departamento de Prática de Saúde Pública da Faculdade de Saúde Pública da USP - Av. Dr. Arnaldo, 715 - São Paulo, S.P., Brasil.

** Do Centro de Dinâmica Populacional (CEDIP) - Faculdade de Saúde Pública da USP - Av. Dr. Arnaldo, 715 - São Paulo, S.P., Brasil. 
CIARI Jr., C. et al. - Avaliação quantitativa de serviços de pré-natal. Rev. Saúde públ., S. Paulo, 6: $361.70,1972$.

tes serviços, pois, torna-se por demais oneroso ao hospital atender complicaçōes obstétricas que poderiam ter sido controladas durante a gestação.

No duplo aspecto de administração em serviços de Saúde Materna e de proteção ao grupo gestante, torna-se necessário desenvolver métodos que permitam a avaliação correta dos serviços, para posterior reprogramação.

Sob o ponto de vista administrativo, a simples verificação do número de consultas não revela efetiva utilização do tempo de serviço profissional. É necessário fazer a avaliação a partir do número de horas de trabalho e do volume de consultas esperadas para o pré-natal, a fim de podermos demonstrar a soma de serviços prestados ao grupo gestante.

Para avaliação do grau de proteção que o pré-natal está conferindo quantitativamente à população de grávidas, são indicadores: a média de consultas por gestante, a média de idade de gestação em que estão sendo feitas as matrículas e a proporção de consultas em relação às matriculadas.

Outro aspecto é a motivação das grávidas para a procura de pré-natal. Alguns fatores sócio-econômico-culturais parecem influir sobre o momento que a mulher decide matricular-se e também determinam sua frequiência ao serviço.

Em trabalho recente ${ }^{2}$, mostramos que o nível de instrução não influi sobre o comparecimento para matrícula nos 3 primeiros meses, igualando-se em todos os graus de conhecimento (analfabeto a universitário). Desta forma, é de supor-se que os serviços complementares existentes no pré-natal, como: curso de preparação ao parto, assistência social, facilidades de internação, de exames de laboratório e medicamentos, ao lado do tipo de atendimento dado pelo pessoal médico e auxiliar, não só determinam o aumento de volume de serviços, como também qualificam as consultas segundo a precocidade da matrícula e constância na frequiência ${ }^{3}$.

Partindo destes pressupostos, pretendeu-se estudar diversos serviços de pré-natal, para estabelecer entre outros dados: 1) curvas de matrícula; 2) proporção de consultas; 3) média de consulta por gestante; 4) média de idade de gestação para matrícula; 5) facilidades oferecidas.

\section{MATERIAL E METODOS}

Foram estudados 10 serviços de pré-natal da Capital e do Interior do Estado de São Paulo, registrando sua qualificação segundo dispusessem de: médicos; enfermeiras; auxiliares de enfermagem; garantia de hospitalização; exames de laboratório; fornecimento de medicamentos; oferta de cursos de preparo ao parto; assistência social de casos.

Em seguida elaborou-se um quadro de registro anual de números de primeiras consultas por idade de gestação e outro com a distribuição de números de consultas por mês de gestação.

Com estes dados, obteve-se a distribuição mensal de matrículas e de consultas subsequientes e seu total anual durante os últimos 4 anos. Registrou-se ainda o tempo de serviço médico.

Para avaliação quantitativa dos serviços prestados referimo-nos aos seguintes indicadores:

1 - média de consultas por médico;

2 - proporção entre consultas dadas e possíveis;

3 - média de idade de gestação na matrícula;

4 - média de consultas por gestante;

5 - proporção entre matriculas e consultas em continuação;

6 - número teórico de consultas;

7 - facilidades oferecidas pelo serviço. 
CIARI Jr., C. et a!. - Avaliaçăo quantitativa de serviços de pré-natal. Rev. Saúde públ., S. Paulo, 6: $361.70,1972$.

MODELO DE FICHA DE REGISTRO

Posto

Matriculas por Idade de Gestação

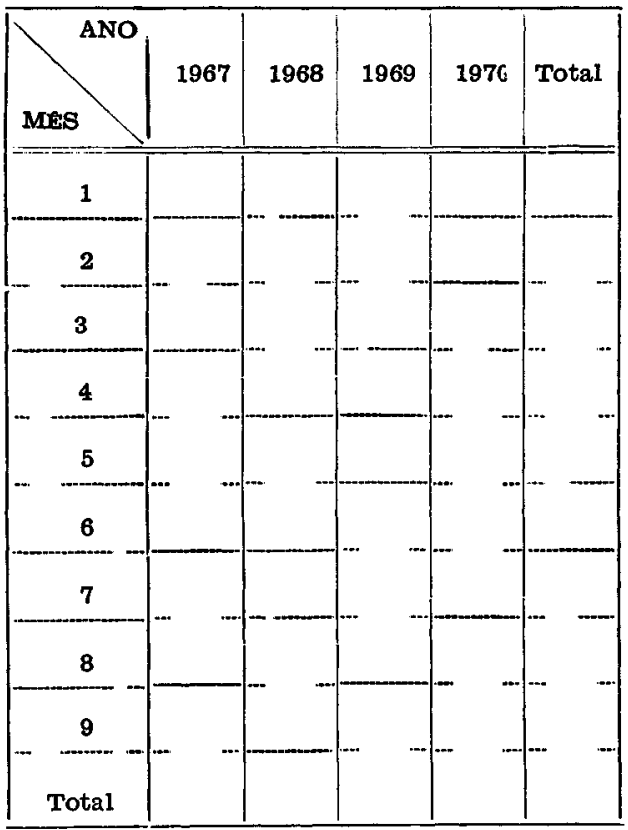

N.o de Médicos/Hora

N.o de Enfermetras

N.o de Auxiliares de Enfermagem

Hospital

\section{1 - Média de consultas por médico.}

É obtida a partir das horas trabalhadas por ano pelo médico. Estas são o produto das horas mensais de trabalho por 11 meses, levando-se em conta as férias. Pode-se ainda deduzir mais ou menos 5 a $10 \%$ das horas, por feriados, faltas, atividades administrativas, etc. Melhor será calcular as horas de trabatho que se costuma avaliar entre 230 a 250 anualmente.

A divisão do número de consultas por ano, por horas trabalhadas nos dá a média de consultas por médico por hora/ano. Por si só esta média significa apenas o trabalho médico executado, e deve ser relacionada ao número de consultas possíveis.
Consultas por mês de Gestação

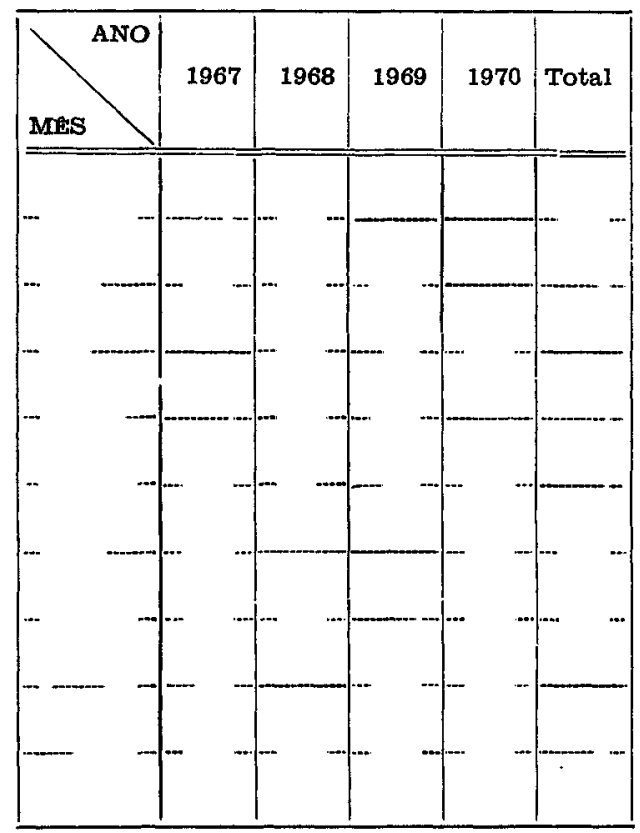

Laboratório

Medicamentos

Cursos

Serviço Social

\section{2 - Nümero de consultas possíveis}

Estas são calculadas multiplicando-se - número de horas/médico/ano pelas consultas habituais por hora. Normalmente este número situa-se, em Saúde Materna entre 4 a 6 , porém, pode variar segundo o critério de registro: apenas para consultas ou para atendimento de todo o tipo (renovação de receitas, informação de exames etc.) 4.

Obtido o número de consultas possíveis, faremos a proporção com aquelas dadas pela fórmula $n d / n p$ onde $n d$ é o número de consultas dadas e $n p$ as possíveis.

Esta proporção nos dá a intensidade 
CIARI Jr., C. et a:. - Avaliaçăo quantitativa de serviços de pré-natal. Rev. Saúde públ., S. Paulo, 6: $361.70,1972$.

ou o que poderemos chamar de consistência do serviço.

\section{3 - Média de idade de gestação na matrícula}

Tanto para o proveito da gestante, quanto para a possibilidade de atuação preventiva, a idade de gestação na matrícula influi decisivamente na assistência. Obtem-se a média da gestação na matrícula por:

$$
\bar{x} \underset{\Sigma}{\sum} \frac{\pi_{i}^{x^{i}}}{i^{i}} \text { onde } x_{i} \text { é a idade }
$$

da gestação e $\mathbf{f}^{\mathbf{i}} \mathbf{o}$ número de matrículas por mês.

4 - Média de consultas por gestante.

E uma medida de frequiência obtida por $n d / n m$ onde $n d$ é o número de consultas dadas e $n m$ o número de matrículas. Isoladamente demonstra o número de atendimentos que cada mulher obtem no serviço. Porém, comparada a outras medidas como número teórico de consultas e proporção de consultas, demonstra o grau de proteção oferecido à gestante. Relaciona-se ainda com a média de idade de gestação na matrícula.

\section{5 - Proporção de consultas.}

Esta pode ser obtida por dois tipos de comparação. Um seria medindo a proporção entre consultas dadas e teóricas, e o outro pela proporção da diferença entre consultas em continuação e matrículas com relação a consultas. $O$ primeiro só deve ser utilizado quando o cálculo de consultas teóricas pode ter uma correção, quanto a abortamentos, prematuros e abandono do serviço.
O segundo foi utilizado por nós, calculando $x-y / x$ onde $x$ é o número de consultas em continuação e $y$ o número de matrículas.

Num serviço ideal, todas as mulheres entrariam para ele no primeiro trimestre e seriam atendidas segundo o número de consultas admitidas pelo serviço, que no nosso caso foi 5 e portanto, a proporção seria: $5 \mathrm{x}-\mathrm{x} / 5 \mathrm{x}=4 \mathrm{x} / 5 \mathrm{x}=$ 0,80 sendo este portanto, o nível ideal de consultas. Em contrapartida, um serviço em que hipoteticamente só houves se a consulta de matrícula teríamos, $x-x / x=0 *$. Desta forma, quando 0 índice for pequeno, menor será a proporção, entre a diferença de consultas e matrículas com relação às consultas.

Para elaboração de nosso gráfico usamos este procedimento, porém, com $\mathrm{x}-\mathrm{y} / \mathrm{x}$ e feito mês a mês.

\section{6 - Nümero teórico de consultas.}

Ao ser matriculada, a mulher passa a ter um número esperado de consultas, segundo a idade de sua gestação, e o número de consultas destinadas pelo serviço a cada gestante. Desta forma, se for aceita como norma, uma consul. ta mensal, a matriculada no $10^{\circ}$ mês terá o número esperado de 9 consultas. Considerando, teoricamente, que nenhuma mulher abandone o serviço, obteremos o total de consultas teóricas a partir do número de matrículas segundo a idade ou número esperado de consultas.

Este número teórico, comparado ao de consultas efetivamente dadas, mede - grau de proteção que o serviço oferece às gestantes. Uma consulta mensal, se bem que desejável, é muito dificilmente obtida 5,6. Desta forma, é preferivel estabelecer a possibilidade de 5

* Para elevarmos o indice 0,80 para 1,0 que é maís comum, poderiamos usar: proporção $=$ consultas-matrículas/ $(n-1)$ consultas onde $n$ é $o$ número de consultas. Donde teriamos: $n x-x(n-1) x=(n-1) x(n-1) x=1$. 
CIARi Jr., C et al. - Avaliação quantitativa de serviços de pré-natal. Rev. Saúde públ., S. Paulo, 6: 361.70 , 1972 .

consultas por gestante (número ainda alto, mas que assegura bom atendimento) assim distribuidas:

- primeiro trimestre 5 consultas

- segundo trimestre 4 consultas

- último trimestre 2 consultas

\section{7 - Qualificação do serviço}

Levamos em consideração os seguintes aspectos:

$$
\begin{aligned}
& \text { a - pessoal } \\
& \text { - médico } \\
& \text { - enfermeira } \\
& \text { - auxiliar de enfermagem } \\
& \text { b - facilidades } \\
& \text { - hospitalização } \\
& \text { - laboratório } \\
& \text { - medicamentos } \\
& \text { - cursos } \\
& \text { - Assistência Social }
\end{aligned}
$$

Para qualificar os serviços utilizamos um critério simples de notas atribuídas por item. O valor da nota vai de 0 a 2 sendo:

\section{0. inexistente}

1. regular

2. bom

\section{RESUITA DOS}

$O$ total de atividades dos 10 serviços observados durante 4 anos está na Tabela 1 , onde vemos o número de matrículas e o número de consultas em continuaçāo. Deles podemos extrair $n m, n d$ e nc, ou seja, número de matrículas, número de consultas dadas e número de consultas em continuação.

Na Tabela 2 temos os valores obtidos nos postos em 4 anos de atividades $e$ que se prestam para os cálculos de avaiação.

Os resultados com referência à média de consultas por hora, proporção entre consultas dadas e possíveis, média de consultas por gestante, proporçāo de matriculas com consultas em continua-

\begin{tabular}{|c|c|c|c|c|c|c|c|c|c|c|}
\hline 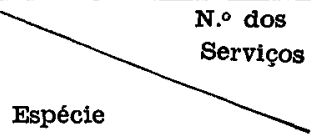 & 1 & 2 & 3 & 4 & 5 & 6 & 7 & 8 & 9 & 10 \\
\hline Matrículas & 589 & 1.517 & 2.650 & 4.097 & 6.609 & 1.166 & 445 & 777 & 2.524 & 256 \\
\hline $\begin{array}{l}\text { Consultas em } \\
\text { continuação }\end{array}$ & 1.637 & 3.317 & 3.307 & 6.336 & 9.094 & 4.706 & 3.408 & 1.527 & 8.644 & 1.627 \\
\hline Total & 2.226 & 4.834 & 5.957 & 10.433 & 15.708 & 5.872 & 3.925 & 2.304 & 11.168 & 1.783 \\
\hline
\end{tabular}
ção, média de idade da gestação na matrícula e qualifıcação dos serviços, encontram-se na Tabela 3.

A distribuição das matrículas por trimestre e sua porcentagem estão na $\mathrm{Ta}$ bela 4 .

T A B EL A 1

Número de matriculas, consultas em continuação e consultas dadas nos 10 serviços em 4 anos 
CIARI Jr., C. et al - Avaliaçăo quantitativa de serviços de pré-natal. Rev. Saúde públ., S. Paulo, 6: $361.70,1972$.

T ABELA 2

Valores de 4 anos de atividades em cada serviço.

\begin{tabular}{rr|r|r|r|r|r|r|r|r|r|r}
\hline $\begin{array}{r}\text { N.o de horas } \\
\text { Serviços }\end{array}$ & 1 & 2 & 3 & 4 & 5 & 6 & 7 & 8 & 9 & 10 \\
\hline
\end{tabular}

N.o de horas trabalhadas por médico/ano.

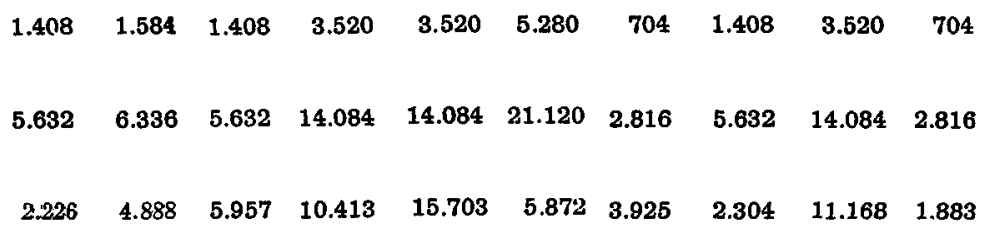

TABELA 3

Resultados dos cálculos de avaliação por serviço nos 4 anos.

\begin{tabular}{|c|c|c|c|c|c|c|c|c|c|c|}
\hline Serviços & 1 & 2 & 3 & 4 & 5 & 6 & 7 & 8 & 9 & 10 \\
\hline $\begin{array}{l}\text { Média de consulta } \\
\text { por hora por } \\
\text { médico }\end{array}$ & 1,58 & 3,08 & 4,23 & 2,96 & 4,46 & 1,11 & 5,57 & 1,63 & 3,17 & 2,67 \\
\hline $\begin{array}{r}\text { Proporção entre } \\
\text { consultas da- } \\
\text { das e possiveis }\end{array}$ & 0,39 & 0,77 & 1,05 & 0,73 & 1,11 & 0,27 & 1,39 & 0,40 & 0,79 & 0,66 \\
\hline $\begin{array}{l}\text { Média de consul- } \\
\text { tas por ges- } \\
\text { tante }\end{array}$ & 3,77 & 3,22 & 2,24 & 2,54 & 2,37 & 5,03 & 8,82 & 2,96 & 4,42 & 7,35 \\
\hline $\begin{array}{l}\text { Proporção de con- } \\
\text { sultas em con- } \\
\text { tinuação c/ma- } \\
\text { trícula. }\end{array}$ & 0,64 & 0,50 & 0,19 & 0,35 & 0,27 & 0,75 & 0,87 & 0,40 & 0,70 & 0,84 \\
\hline $\begin{array}{l}\text { Média de idade } \\
\text { da gestaçăo na } \\
\text { matrfcula }\end{array}$ & 4,86 & 5,09 & 6,78 & 6,59 & 6,41 & 4,45 & 3,18 & 4,86 & 4,86 & 3,27 \\
\hline $\begin{array}{l}\text { Quallficaçăo dos } \\
\text { serviços }\end{array}$ & 4 & 4 & 5 & 5 & 5 & 13 & 16 & 4 & 10 & 10 \\
\hline
\end{tabular}


CIARI Jr., C. et al. -- Avaliação quantitativa de serviços de pré-natal. Rev. Saúde públ., S. Paulo, 6: 361.70, 1972.

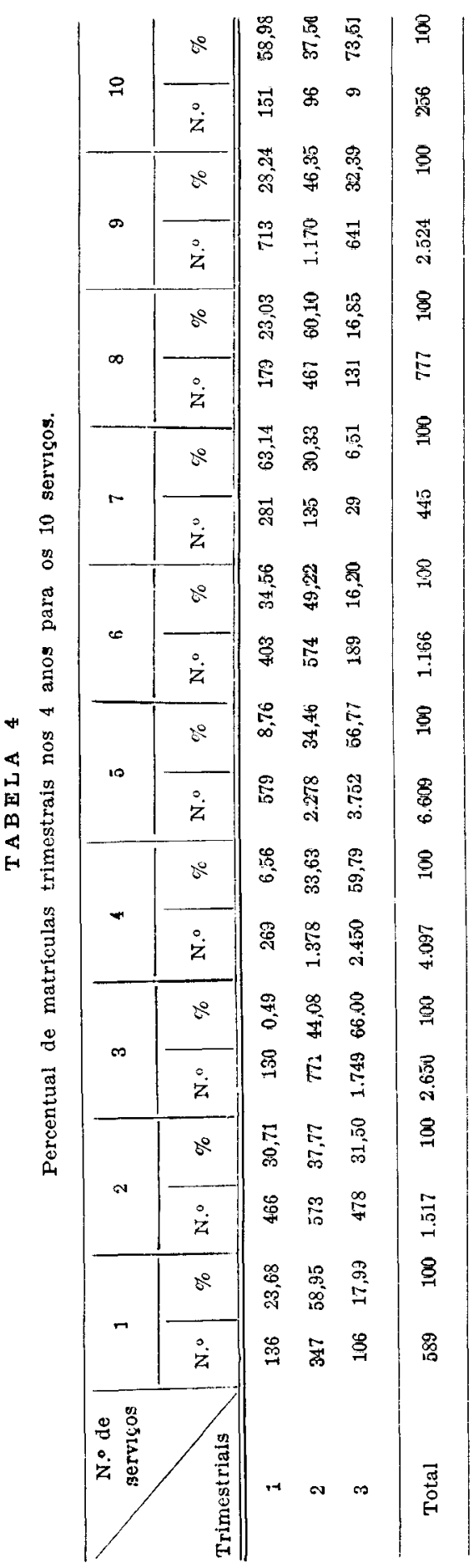

As Figuras 1, 2, e 3 são exemplos da forma que assume a distribuição das matrículas mensais e por trimestre e proporção de consultas em serviços com caracteristicas de matrícula normal (Figura 1), com predominância do $20^{\circ}$ trimestre (Figura 2) e com predominância no $3 .^{\circ}$ trimestre (Figura 3).

Temos na abscissa os meses e na ordenada a porcentagem.

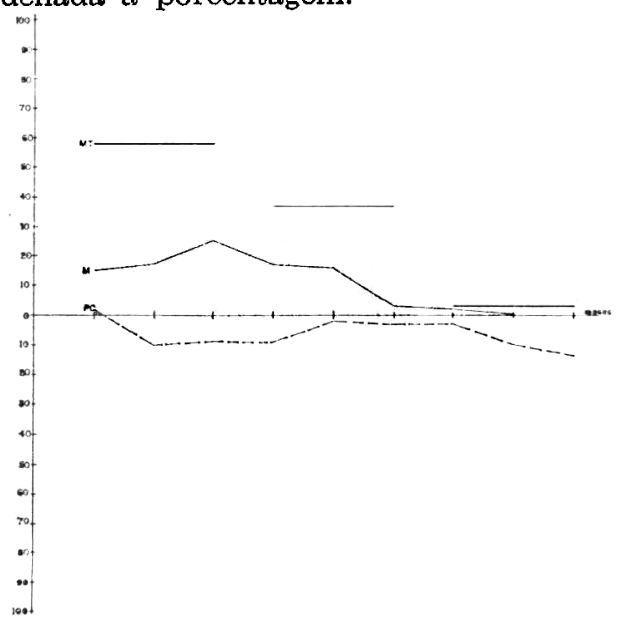

Figura 1 - Distribuição de matrículas trimestrais e mensais e proporção de consul. tas matrículas trimestrais (Me consultas (PC).

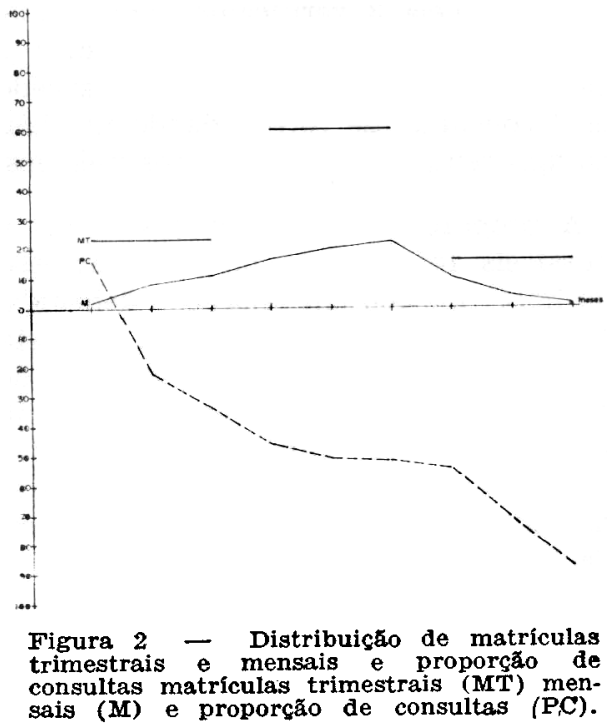


CIARI Jr., C, et al. - Avaliação quantitativa de serviços de pré-natal. Rev. Saúde públ., s. Paulo, 6: $361: 70$, 1972 .

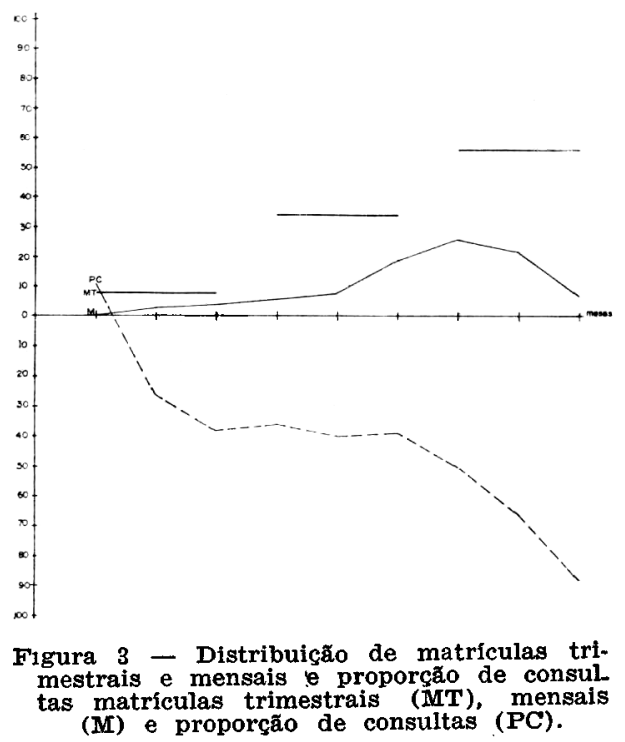

D I S C U S S O

Nos 10 serviços por nós observados durante os 4 anos de funcionamento, não houve variações significativas de atividades; portanto, podemos tomar como base dos cálculos a soma total de serviços nesses anos.

Pela Tabela 3 verificamos que a média horária de consultas por médico é baixa. Apenas 3 delas atinge ou excede as 4 consultas de rotina, dando um baixo aproveitamento dos serviços médicos.

A proporção entre consultas dadas e possíveis revela mais uma vez excesso de horas/médico em relação à demanda. Vemos que em 7 dos 10 locais ela está abaixo de um, que seria a situação de equilíbrio. Como era de esperar-se o nível da proporção entre consultas dadas e possíveis acompanha a média horária de consultas por médico, pois elas interdependem.

Estas duas medidas podem ser consideradas como indicadores quantitativos da atividade médica. De certa forma, re- flete-se na qualidade dos serviços, pois, se de um lado é conveniente não sobrecarregar o profissional com alto número de consultas, por outro, uma quantidade muito pequena demonstra falta de assistência à gestante.

A média de consultas por gestante é indicador da intensidade com que as mulheres são assistidas. Sabemos ser impossivel dar adequada cobertura em pré-natal, com menos de 4 consultas por gestante. Verificamos na Tabela 3 que apenas 4 serviços ultrapassam este valor.

Um indicador significativo das atividades de pré-natal é a proporção entre as matrículas e as consultas em continuação, pois estas representam a assistência que efetivamente está sendo oferecida à mulher. Mede também o grau de interesse revelado pelas pacientes, na proteção de sua saúde, pois se relaciona à freqüência mantida por elas. Nos serviços por nós observados vemos que apenas dois ultrapassam o índice 0,80 mantendo-se os demais em nivel inferior e alguns bastante baixos. Isto nos mostra a pequena proteção que está sendo oferecida às mulheres frequientes a estes serviços.

Outro aspecto de medida importante para analisar não só a quantidade de serviços, como para qualificá-los, é a média de idade de gestação com que as mulheres estão se matriculando. Ela nos mostra a possibilidade de proteção que pode ser oferecida à gestante, pois sabemos que as matriculas tardias dificultam a assistência.

Não é de esperar-se número significativo de matrículas no primeiro trimestre, pois CIARI $^{2}$ demonstrou que mesmo mulheres com alto nível intelectual dirigem-se a serviços pré-natal mais tardiamente. No entanto, dependendo da precocidade da matrícula neste trimestre, podemos ainda obter média de idade 
CIARI Jr., C. et al. - Ávaliação quantitativa de serviços de pré-natal. Rev. Saúde públ., S. Paulo, 6: $361.70,1972$.

mais baixa. Nos serviços observados confirma-se a tendência para $020^{\circ}$ trimestre, porém, alguns deles, com maior número no final, elevando assim a idade de gestação na matrícula, o que prejudica a assistência à gestante.

Por último, acreditava-se que a qualificação do serviço ou as facilidades que ele oferece são elementos decisivos para melhorar a frequiência. $O$ que notamos é que isto só tem valor quando representa uma soma grande de benefícios à gestante, como é o caso do serviço n. 7 . o fornecimento de alguma vantagem pode até distorcer a frequiência, como nos de n.'s 3, 4 e 5, onde a obtenção de guia para a hospitalização, fornecida a qualquer tempo, determina uma procura nas idades finais, como vemos pela média de idade de gravidez na matrícula.

As Figuras 1, 2 e 3 que apresentam três tipos de serviços, são ilustrativos para demonstrar o comportamento de matrículas mensais e trimestrais e a curva de proporção de consultas. A Figura 1 é o representativo de serviços onde notamos distribuição ideal das matriculas trimestrais e consequiente aproximação. Na Figura 3, já vemos o inverso com típica variação entre matriculas por mês e consultas. Finalmente, a Figura 2 representa os serviços mais comuns com a concentração de matrículas no $2^{\circ}$ trimestre, mas sempre com discrepância entre matrículas e consultas em continuação.

\section{CONCLUSOES}

1 - Nos 10 serviços observados, verifica-se que a maioria revela acentuado tempo ocioso dos profissionais médicos, demonstrado não só pelo número médio de consultas por hora como também pela relação de proporção entre consultas dadas com as possíveis.

2 - A cobertura assistencial as mulheres medida pela média de consultas por gestante e proporção entre consul- tas dadas e teóricas está baixa, se considerarmos como mínimo 4 consultas por grávida.

3 - Esta proteção está prejudicada, se levarmos em consideração a idade de gestação em que é iniciada a assistência prénatal, pois, apenas em dois serviços, as matrículas se concentraram próxima ao $10^{\circ}$ trimestre de gravidez. Não parece existir uma relação global entre os benefícios oferecidos pelo serviço e a frequiência.

4 - Os sete itens de avaliação propostos parecem ser indicadores da atividade quantitativa $e$ indiretamente permitem avaliar a qualidade dos serviços.

RSPSP-149

CIARI JR., C. et al. - [Quantitative evaluation of the activities of prenatal services]. Rev. Saúde públ., S. Paulo, 6:361-70, 1972.

Summary: Six items are proposed in order to obtain a quantitative evaluation of the activities in the prenatal services. Indexes of phyhicians' activities and degree of protection atainned by patients are: 1) the number of consults by doctorhourfyear; 2) the number of possible consults; 3) the mean number of consults by pregnants; 4) the ratio between registrations and consults (other than the first) by month; 5) mean age of pregnancy at the time of registration. These data are examined in relation to the quality of the services, $i$. e., doctor's presence, nurses, internation facilities, laboratory, prescriptions, courses and social care. It seems that those indexes exhibit not only the quantity of services offered but as well, their quality. Ten prenatal services were observed during $a$ period of 4 years. Their activities were evaluated through the indexes mentioned, and a low productivity was found in their majority, with too high number of vacant hours and a small number of consults. The assumed relationship between quality of services and $a$ higher prenatal center attendance is not clear, unless where the service offer is intensive.

UNITERMs: Prenatal care * Pregnancy *. 
CIARI Jr., C. et al. - Avaliação quantitativa de serviços de pré-natal. Rev. Saúde públ., S. Paulo, 6: 361.70, 1972 .

\section{REIPERNCIAS BIBLIOGRAFICAS}

1 - ANTOINE, M. J. - Report of one year prenatal and post natal consultations at the "Centre de Protection Maternalle et Infantile et d'Education Sanitary". Bull. zTed. Obst. franc., 20:1-14, 1968 .

2 - CIARI Jr., C. \& ALMEIDA, P. A. M. de Elementos de avaliação do "risco gravi. dico". Rev. Saride públ., S. Paulo, 6:57-78, 1972 .

3-GARDINER, S. H. - Motivation for obstetric care. Obst. Gynec., 33:306-12, 1969.

4-.INFORME sobre el Progresso de la In vestigation Interamericana de Morta lidad en la Niñes. Washington, D.C. Organizacion Panamericana de La
Salud, 1970 [Apresentado a la 9.a reunión del Comité Asesor de Investigacion Médica de la OPS/OMS 1970].

5 - ORganizaÇAO MUNDIAL DA SAUDE. Comité d'Experts de l'Hygiene de la Maternité et de l'Enfance, Genéve, 1955. L'administration des services d'hygiene de la Maternité et de l'enfance; 2 eme rapport. Genéve, 1957. (Ser. Rapp. Tech., 115).

6 - ORGaNizaçÃo MUNDIAL DA SAUde. Comité de Expertos de la OMs en Higiene Maternoinfantil. Ginebra. 1968. Organizacion y administracion de los Servicios de higiene maternoinfantil: 5. informe. Ginebra, 1969. (Ser. Inf. Tesh., 428).

Recebido para publicą̧ão em 2-10-1972

Aprovado para publicagão em 25-10-1972 